\title{
Self-avoiding linear and star polymers anchored to membranes
}

\author{
Thorsten Auth and Gerhard Gompper \\ Institut für Festkörperforschung, Forschungszentrum Jülich, D-52425 Jülich, Germany \\ (Received 25 March 2003; revised manuscript received 17 July 2003; published 7 November 2003)
}

\begin{abstract}
The effect of anchored linear and star polymers in the mushroom regime on the curvature elasticity of membranes is investigated by Monte Carlo simulations and scaling arguments. We describe a simulation method to calculate the free energy of anchored polymers as a function of membrane curvature, which is accurate enough to extract reliable values for the polymer-induced spontaneous curvature $\Delta c_{0}$, bending rigidity $\Delta \kappa$, and saddle-splay modulus $\Delta \bar{\kappa}$. For self-avoiding linear and star polymers, the universal amplitudes of the curvature moduli as well as the effects of finite chain lengths are determined, to our knowledge, for the first time. We find that star polymers have the unique property of strongly affecting $c_{0}$ and $\kappa$, but leaving $\bar{\kappa}$ essentially unchanged. Furthermore, star polymers are shown to have a much stronger effect on membrane properties than an equivalent number of linear polymers.
\end{abstract}

DOI: 10.1103/PhysRevE.68.051801

PACS number(s): 36.20.-r, 87.16.Dg, 82.70.-y

\section{INTRODUCTION}

The shapes, fluctuations, and phase behavior of fluid membranes are controlled by their curvature elasticity [1]. The bending rigidity $\kappa$, the saddle-splay modulus $\bar{\kappa}$, and the spontaneous curvature $c_{0}$ are strongly affected by the composition of the membrane, and by the interaction of the membrane with the surrounding fluid and with macromolecules and ions embedded in it. From a theoretical point of view, the interaction of membranes with embedded [2], anchored [3-7], adsorbed [8-10], or free [11,12] polymers is particularly interesting, because due to the dominance of entropical contributions to the free energy of these macromolecules, their effect on the curvature elasticity depends not on the detailed chemical structure, but only on a few geometrical parameters such as the polymer length. Similarly, the electrostatic contribution to the curvature elasticity of charged membranes only depends on the surface charge density, the Debye screening length, and the Bjerrum length [13,14]. Experimentally, membranes decorated with anchored polymers have been studied for end-grafted polymers at bilayer vesicles $[15,16]$ and in lamellar hydrogels [17], for symmetric and asymmetric amphiphilic block copolymers in oilwater-surfactant microemulsions [18-20], for hydrophobically modified polymers in surfactant lamellar phases [21], and for symmetric amphiphilic triblock copolymers in bilayer lyotropic phases [22]. All these experiments are performed in good solvent conditions for the polymers. It is therefore important to understand the effect of the excludedvolume interaction of the polymers on the membrane properties.

In all the theoretical results mentioned above, the ratio of the additional contribution to the bending rigidity and to the saddle-splay modulus is a constant, i.e., $\kappa$ and $\bar{\kappa}$ are affected in exactly the same way. Furthermore, these constants are all in the range $-0.3>\kappa / \bar{\kappa}>-2.2$. For example, for ideal polymers anchored to a membrane in the mushroom regime of low grafting density, $\kappa / \bar{\kappa}=-(1 / 2+\pi / 4)=-1.285[5,11]$, independent of chain length $N$, while for polymer brushes at high grafting density, $\kappa / \bar{\kappa}=-(2+\nu) /(2 \nu)=-13 / 6=$ -2.167 (with $\nu=3 / 5$ ) from scaling theory [5] and $\kappa / \bar{\kappa}=$
$-105 / 64=-1.641$ from mean-field theory [3], independent of both chain length and grafting density. For polymers in dilute solution with excluded-volume interactions, $\kappa / \bar{\kappa}=$ $-(1+0.10 \epsilon)$ in $d=4-\epsilon$ spatial dimensions [12]. Similarly, for polymers embedded in the membrane, $\kappa / \bar{\kappa}=-1 / 2$ [2]. Finally, charged membranes in the limit of low surface charge density have $\kappa / \bar{\kappa}=-3 / 2$, while for membranes at high charge density, $\kappa / \bar{\kappa}=-3 / \pi^{2}=-0.304[14]$.

In this paper, we show that in the case of star polymers anchored to a membrane at their center, the dependence of the bending rigidity and the saddle-splay modulus on the functionality $f$ (the number of arms) is different. Thus, star polymers provide a unique opportunity to control the bending rigidity and the saddle-splay modulus independently. Furthermore, we show that star polymers are more efficient in affecting the curvature elasticity of membranes than linear polymers in the mushroom regime. Finally, we calculate the universal amplitudes of all three elastic parameters $\kappa, \bar{\kappa}$, and $c_{0}$ for self-avoiding linear and star polymers, to our knowledge, for the first time.

\section{MODEL AND SIMULATION TECHNIQUE}

The calculation of curvature elasticities from Monte Carlo simulations in the mushroom regime is very difficult, since the polymer effect is only of the order of the thermal energy $k_{B} T$, even close to the overlap grafting density. In addition, the curvature elastic moduli follow from the minute differences in the polymer free energies at a planar and a curved wall in the limit of small curvatures.

We have therefore devised the following method, which is both efficient and sufficiently accurate to obtain reliable data. The main idea is to run a simulation of a polymer anchored to a planar, hard wall. We use the standard pivot algorithm [23] to perform this simulation. For each configuration, we construct a sphere of radius $R_{s}$ and a cylinder of radius $R_{c}$ in such a way that (i) it touches the wall at the anchoring point, (ii) all monomers are inside it, and (iii) the radii $R_{s}$ and $R_{c}$ are minimal, as illustrated in Fig. 1. The cylinder axis is always taken to have the same, fixed orientation. The ratio of partition functions, $Z_{s}\left(R_{s}, N\right) / Z_{p}(N)$, of a polymer confined 


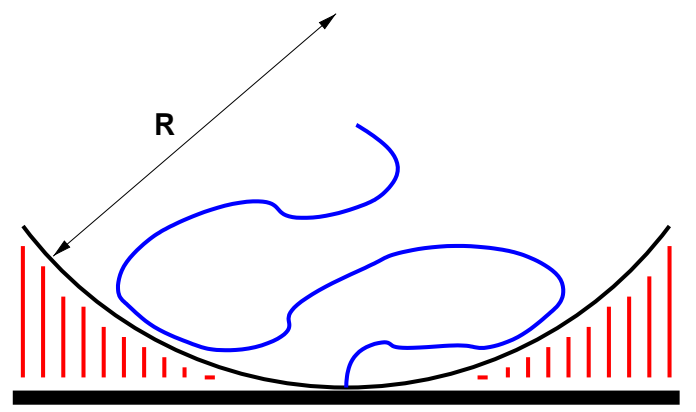

FIG. 1. A polymer anchored to a planar, hard wall is simulated. Enveloping spheres and cylinders of radius $R$ are constructed to calculate the ratio of partition functions $Z(R, N) / Z_{p}(N)$.

to the inside a sphere of radius $R_{s}$ and a planar hard wall is then obtained by simply counting the fraction of all configurations, for which all monomers fall inside the sphere. The same method gives the ratio of partition functions, $Z_{c}\left(R_{c}, N\right) / Z_{p}(N)$, for the cylinder. One of the big advantages of this method is that the partition functions for all radii of curvature can be obtained from a single run.

The method described so far seems to be restricted to membranes curved towards the polymer chain. However, it can easily be generalized to membranes curving away from the polymer. All what has to be done in the latter case is to simulate a polymer anchored at the outside of a sphere of some fixed radius $R_{0}$. The same technique described above of constructing enveloping spheres and cylinders with radii $R_{s}, R_{c}>R_{0}$ then allows to calculate the ratios of partition functions $Z_{s}\left(-R_{s}, N\right) / Z_{p}(N)$ and $Z_{c}\left(-R_{c}, N\right) / Z_{p}(N)$. Here, the normalization factor $Z_{s}\left(-R_{0}, N\right) / Z_{p}(N)$ is obtained automatically by using the plane as enveloping surface.

As in the analytical approaches, the ratio of partition functions is expanded in powers of inverse radius of curvature,

$$
\begin{aligned}
& \frac{Z_{c}(R, N)}{Z_{p}(N)}=1+a_{1} \frac{R_{e}}{R}+a_{2}\left(\frac{R_{e}}{R}\right)^{2}+\cdots, \\
& \frac{Z_{s}(R, N)}{Z_{p}(N)}=1+b_{1} \frac{R_{e}}{R}+b_{2}\left(\frac{R_{e}}{R}\right)^{2}+\cdots,
\end{aligned}
$$

where $R_{e} \sim N^{\nu}$ is the average end-to-end distance of a free polymer, with $\nu=0.59$ in good solvent and $\nu=1 / 2$ in a theta solvent, respectively. Consistency with the curvature energy requires $b_{1}=2 a_{1}$. The simulated partition function ratios as well as large- $R$ expansions extracted from the data up to second order are plotted in Fig. 2. This figure also demonstrates nicely that the expansions hold for curvatures of both signs which is a necessary prerequisite to describe the polymer effect by changes of the curvature elastic constants. We have shown that Eqs. (1) and (2) are valid for all linear and star polymers considered in this paper [24]. The elastic constants (in units of $k_{B} T$ ) are finally obtained as

$$
\begin{gathered}
\kappa_{e f f} \Delta c_{0}=a_{s p} \sigma R_{e}, \\
\Delta \kappa=a_{\kappa} \sigma R_{e}^{2},
\end{gathered}
$$

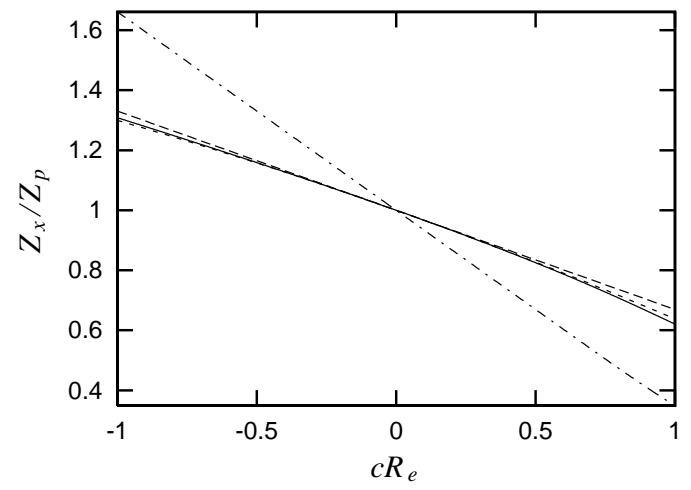

FIG. 2. Simulation results for the partition-function ratios of an ideal polymer chain with 20 bonds. Contributions to the smallcurvature expansion up to quadratic order are used to evaluate the effect on the membrane parameters. In the sphere case, $Z_{s}(R, N) / Z_{p}(N)$, all terms of higher than linear order vanish; fit and simulation curve match exactly (dashed-dotted line). In the cylinder case, $Z_{c}(R, N) / Z_{p}(N)$, simulation data (solid), linear (long dashes), and linear plus quadratic contributions (short dashes) are shown.

$$
\Delta \bar{\kappa}=\bar{a}_{\kappa} \sigma R_{e}^{2}
$$

where $\sigma$ is the grafting density of the polymer chains, $\kappa_{e f f}$ the bending rigidity of membrane plus anchored polymers, and $a_{s p}=-a_{1} / 2, a_{\kappa}=\left(2 a_{2}+a_{1}^{2}\right), \bar{a}_{\kappa}=\left(b_{2}-4 a_{2}\right)$.

\section{IDEAL AND SELF-AVOIDING LINEAR POLYMERS}

We have tested the method for a single, anchored, ideal polymer chain, for which analytical results are available in the long-chain limit [5]. We employ the standard freely jointed chain model with fixed bond length. In order to obtain data of sufficient statistical accuracy, Monte Carlo runs of typically $10^{8}$ million Monte Carlo steps per bead are required. The studied chain lengths range from $N=10$ to $N$ $=200$.

It is immediately clear that we have to do a double extrapolation in order to compare our data with the analytical results. First, we have to extrapolate from finite curvatures to the limit of (almost) vanishing curvature, because the relative statistical errors of the simulation data diverge in the limit $R \rightarrow \infty$. This is done by fitting a quadratic polynomial to the data for $1-Z_{x}\left(R_{s}, N\right) / Z_{p}(N)$ with $x=s, c$ in the range 0 $<1 / R<c_{\text {max }}$, in which the data are not yet strongly affected by higher-order terms in the curvature expansion. The resulting coefficients $a_{1}, a_{2}, b_{1}$, and $b_{2}$ still have a weak dependence on $c_{\max }$. We extrapolate to $c_{\max }=0$ by fitting the data for $a_{1}\left(c_{\max }\right)$ etc. again to a linear or quadratic polynomial, ignoring the data for very small $c_{\text {max }}$, which has large statistical error bars. Second, we have to extrapolate to large chain lengths $N \rightarrow \infty$, since the analytical results are obtained in the continuum limit. A plot of the data for the bending rigidity versus $N^{-1 / 2}$ shows a nearly linear dependence, compare Fig. 3 so that the correction-to-scaling exponent must be close to $1 / 2$. This should be compared with very similar values for the correction-to-scaling exponent in the hydrodynamic radius of ideal chains [25] and for the end-to-end dis- 


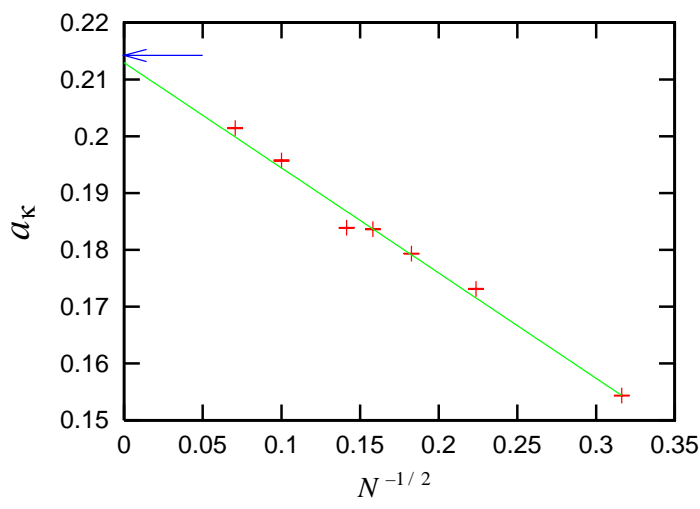

FIG. 3. The universal amplitude $a_{\kappa}$ of the bending rigidity of an ideal polymer chain of $N$ monomers. The arrow indicates the analytical result $[5,11]$.

tance of self-avoiding chains [26]. Also, a self-consistentfield calculation of the effect of long block copolymers on the curvature elasticity of interfaces in a ternary mixture of two homopolymers and a short block copolymer is consistent with this correction-to-scaling exponent [27]. A linear fit to the data implies

$$
a_{\kappa}=0.2130\left(1-0.870 N^{-1 / 2}+\cdots\right) .
$$

A comparison with the analytical result $a_{\kappa}=(1+\pi / 2) / 12$ $=0.2142$ shows excellent agreement, which demonstrates that our method works very well. Furthermore, Eq. (6) indicates that corrections to scaling can be quite large for the typical chain lengths used in experiments, and that they reduce the polymer effect. The results for the spontaneous curvature and the saddle-splay modulus are

$$
\begin{aligned}
& a_{s p}=0.1801\left(1-0.369 N^{-1 / 2}+\cdots\right), \\
& \bar{a}_{\kappa}=-0.1682\left(1-1.179 N^{-1 / 2}+\cdots\right)
\end{aligned}
$$

for which the same remarks apply as for $a_{\kappa}$. We estimate the statistical error of all these amplitudes to be about 0.001 .

The method can now be applied with confidence to anchored self-avoiding linear and star polymers. We use the standard model for self-avoiding polymers with hard spheres of radius $r_{0}$ and bonds of fixed length $\ell_{0}$ between neighboring monomers. In order to have a scaling regime as large as possible, we chose $\ell_{0} / r_{0}=4$, for which the end-to-end distance scales as $N^{\nu}$ with $\nu=0.59$ for chain lengths as small as $N=10$. The simulations are again performed with the pivot algorithm [23].

For a self-avoiding linear chain we obtain

$$
\begin{aligned}
& a_{\kappa}=0.1997\left(1-0.9719 N^{-1 / 2}+\cdots\right), \\
& a_{s p}=0.1679\left(1-0.3911 N^{-1 / 2}+\cdots\right), \\
& \bar{a}_{\kappa}=-0.1532\left(1-1.2206 N^{-1 / 2}+\cdots\right)
\end{aligned}
$$

for $N$ monomers, see also Fig. 4. We estimate the statistical error of to be about 0.001 .

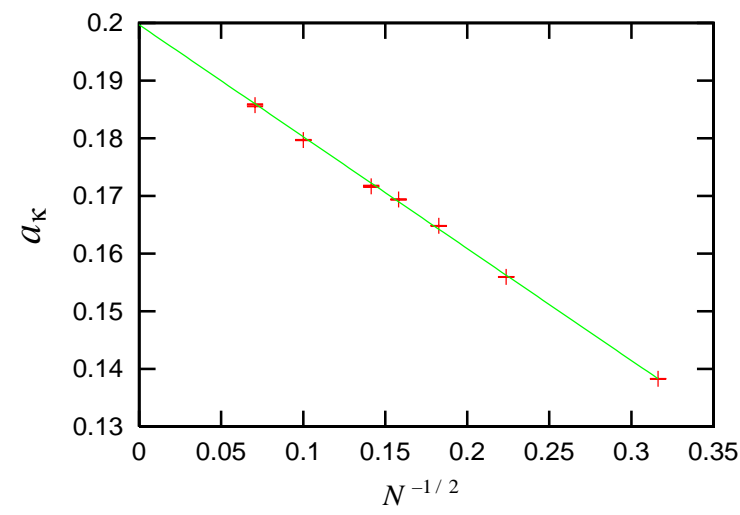

FIG. 4. The universal amplitude $a_{\kappa}$ of the bending rigidity of a self-avoiding polymer chain of $N$ monomers.

Thus, we find that the universal amplitude of the bending rigidity for a linear self-avoiding polymer is $a_{\kappa}=0.1997$ \pm 0.001 , which differs not too much from the result for an ideal chain with $a_{\kappa}=0.2142$. This is indeed a surprising result, since the repulsion between the monomers of a selfavoiding chain leads to a stronger pressure of the polymer onto the membrane compared to the case of an ideal chain [28]. Therefore, a larger amplitude for the self-avoiding chain might be expected. On the other hand, a smaller value of the universal amplitude $a_{\kappa}$ for self-avoiding chains is not inconsistent with an increased pressure, since the decrease of the amplitude is overcompensated by the increase of the endto-end distance. Finally, analytical results for the effect of polymers in solution on the bending rigidity imply $a_{\kappa}($ self-avoiding $) / a_{\kappa}($ ideal $)=1-0.0713 \epsilon$ in $d=4-\epsilon$ spatial dimensions, which is an effect of similar (small) magnitude [12]. In both cases, self-avoidance decreases the magnitude of the universal amplitude. The effects on $c_{0}$ and $\bar{\kappa}$ are similar, compare Fig. 5 for $f=1$ and Table I.

\section{STAR POLYMERS}

Star polymers are modeled as $f$ freely jointed chains with mutual avoidance and self-avoidance, which are all anchored to the same point on the membrane. The results for the spontaneous curvature, the bending rigidity, and the saddle-splay modulus per arm, i.e., the effect of a star polymer divided by the functionality $f$, are shown in Fig. 5 as function of the arm number. This shows immediately two qualitative effects. First, the bending rigidity and the spontaneous curvature per arm increase with $f$. The star polymer is therefore more efficient in modifying these membrane properties than an equal number of individual chains. Second, the saddle-splay modulus per arm remains essentially constant, i.e., the saddlesplay modulus has a different $f$ dependence than the bending rigidity.

The increased efficiency of an $f$-armed star polymer compared to $f$ single polymer chains can be understood intuitively as follows. Consider a symmetric case, in which two identical star polymers are attached on either side of the membrane, so that the spontaneous curvature vanishes identically. The star polymers then impose an entropic pressure 

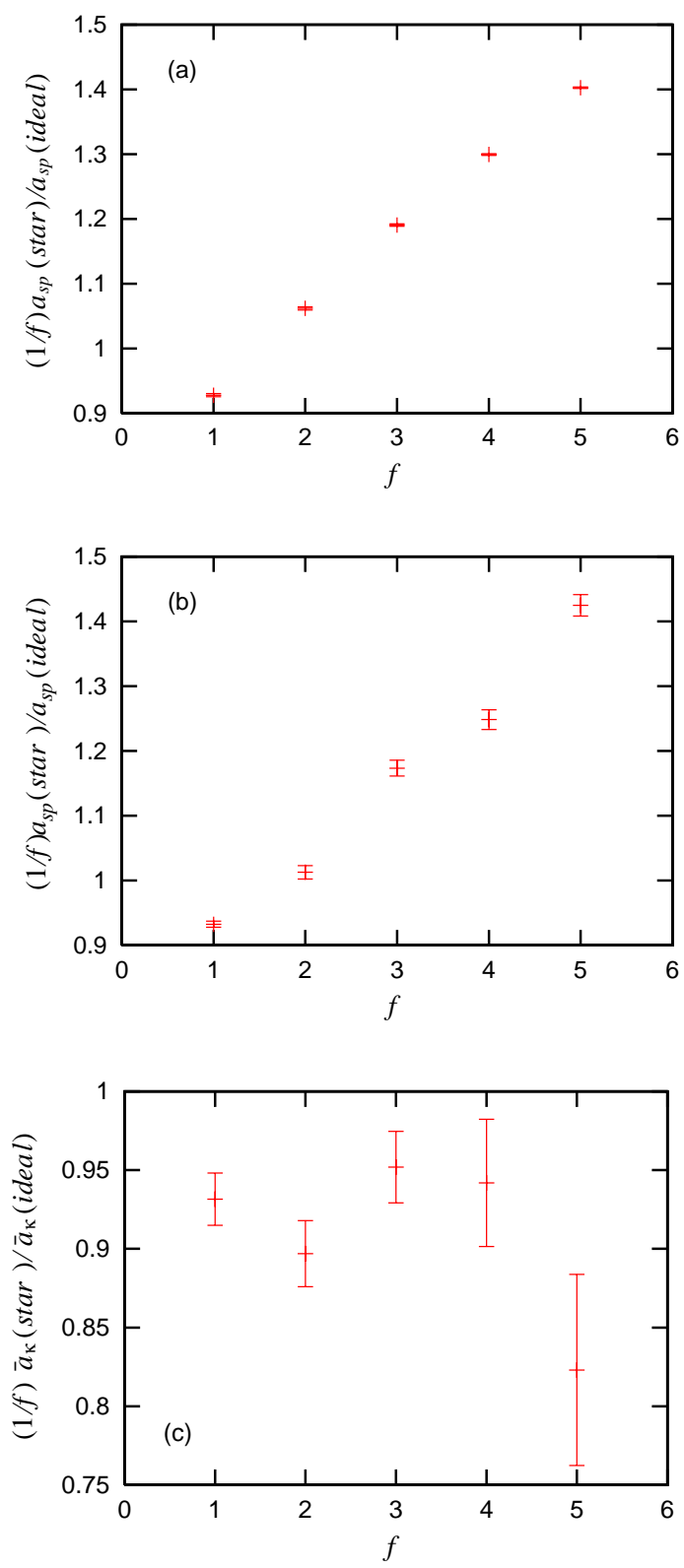

FIG. 5. Effect per arm of an anchored star polymer on the membrane elasticity as a function of the functionality $f$, relative to a star without self-avoidance: universal amplitude (a) $a_{s p}$ of the spontaneous curvature, (b) $a_{\kappa}$ of the bending rigidity, and (c) $\bar{a}_{\kappa}$ of the saddle modulus.

onto the membrane from both sides. When the membrane is curved, the star on the interior side is compressed, while the star in the exterior is relaxed. The same also happens for a linear polymer. However, due to the mutual excluded-volume interactions of the arms of a star polymer, the entropic pressure is higher for the star polymer. We obtain the important quantitative result that the magnitude of the polymer effect per arm on $\kappa$ and $c_{0}$ increases by about a factor 1.5 for $f$ $=5$ compared to single chains, and should reach a factor 2 roughly at $f=10$.

Our results for the two-armed star can be compared with the approximate calculation of Ref. [6], in which the excluded volume was mimicked by an impenetrable plane be-
TABLE I. Universal amplitudes of the contributions of linear anchored polymers on the membrane elasticity. The statistical error in the simulation results is about 0.001 for ideal and self-avoiding chains.

\begin{tabular}{lccc}
\hline \hline & \multicolumn{2}{c}{ Ideal chain } & Self-avoiding \\
\hline & Analytical & Simulation & Simulation \\
$a_{s p}$ & $\sqrt{\pi} /(4 \sqrt{6})$ & +0.1801 & +0.1679 \\
$a_{\kappa}$ & $(1+\pi / 2) / 12$ & +0.2130 & +0.1997 \\
$\bar{a}_{\kappa}$ & $-1 / 6$ & -0.1682 & -0.1532 \\
\hline \hline
\end{tabular}

tween two ideal polymers. This leads to the estimate $\kappa_{e f f} \Delta c_{0}=(7 / 2)[\sqrt{\pi} /(4 \sqrt{6})] \sigma R_{e}$, which overestimates the effect compared to our results by a factor of about 1.6. The reason for this overestimation is clearly that the typical conformations of two chains with excluded-volume interactions show considerable interpenetration, which is not taken into account in the impenetrable-wall approximation.

With increasing functionality, Monte Carlo simulations become less and less efficient. We therefore use the blob model for large functionality $f$, which has been employed before for free star polymers [29], for polymer brushes to calculate the polymer effect on the curvature elasticity $[3,5,7]$, and for anchored star polymers to determine the local membrane shape [30]. The main idea is that at a given distance from the anchoring point, the blob size $\xi$ is determined by the area available to the polymer chains. For a spherical deformation, this is the part of the surface of a sphere of radius $r$ around the anchoring point, which is inside another sphere of radius $R$ (the radius of curvature) touching the wall at the anchoring point. In the case of a cylindrical deformation, the second sphere has to be replaced by a cylinder of radius $R$. This implies

$$
\xi^{2}(r)=\frac{1}{f} \int_{0}^{2 \pi} d \varphi \int_{\theta_{\min }}^{\pi / 2} d \theta r^{2} \cos \theta,
$$

where the angle $\theta_{\min }$ is determined by $\sin \theta_{\min }=r /(2 R)$ for a spherical and by $\sin \theta_{\min }(\varphi)=\left[R-\left(R^{2}-r^{2} \sin ^{2} \varphi \cos ^{2} \varphi\right)^{1 / 2}\right] /$ $\left(r \cos ^{2} \varphi\right)$ for a cylindrical deformation. To relevant order in an expansion in $r / R$, we obtain

$$
\xi^{2}(r)=\frac{2 \pi r^{2}}{f}\left(1-q \frac{r}{R}\right)
$$

with $q=1 / 2$ and $q=1 / 4$ for sphere and cylinder, respectively. The number of monomers in a blob at distance $r$ is then given by $n(r)=\left[\xi(r) / \ell_{0}\right]^{1 / \nu}$. The total number of monomers in one chain is

$$
N=\int_{0}^{R_{\text {star }}} d r \frac{n(r)}{\xi(r)},
$$

which determines the radius $R_{\text {star }}$ of the star polymer. Expanding to second order in $1 / R$, we find

$$
R_{\text {star }}=f^{(1-\nu) / 2} \ell_{0} N^{\nu} \Gamma\left(f^{(1-\nu) / 2} \frac{\ell_{0} N^{\nu}}{R}\right)
$$


with a scaling function $\Gamma(x)=Q_{0}+Q_{1} x+Q_{2} x^{2}+\ldots$ and positive constants $Q_{0}, Q_{1}$, and $Q_{2}$, where the latter two depend on the (spherical or cylindrical) type of deformation. Equation (15) shows that the star radius increases with the functionality [29]. More importantly in our case, Eq. (15) demonstrates explicitly that the radius of a star in the inside (outside) of a curved membrane increases (decreases) with the scaled curvature $R_{\text {star }}^{(0)} / R$, where $R_{\text {star }}^{(0)}=f^{(1-\nu) / 2} \ell_{0} N^{\nu}$ is the star radius at a planar wall. Thus, the arms have to stretch more at higher functionality, which implies a larger effect on $c_{0}$ and $\kappa$.

Finally, the free energy of one chain is determined by

$$
\mathcal{F}_{1}=k_{B} T \int_{\ell_{0}}^{R_{s t a r}} d r \frac{1}{\xi(r)}
$$

By evaluating these integrals in an expansion in $1 / R$ for both sphere and cylinder, we can extract the contribution of the star polymer to the curvature elasticity. The result of this calculation is

$$
\begin{gathered}
\kappa_{e f f} \Delta c_{0}=A_{s p} f^{2-\nu / 2} \sigma \ell_{0} N^{\nu}, \\
\Delta \kappa=A_{\kappa} f^{5 / 2-\nu} \sigma \ell_{0}^{2} N^{2 \nu}, \\
\Delta \bar{\kappa}=0,
\end{gathered}
$$

where $A_{s p}=0.029$ and $A_{\kappa}=0.110$ for $\nu=0.59$. Thus, the elastic moduli per arm are found to increase with the functionality as $\Delta \kappa / f \sim f^{0.91}$ and $\kappa_{e f f} \Delta c_{0} / f \sim f^{0.71}$. These power laws for large $f$ with exponents not too far from unity are very consistent with the almost linear dependence found in the simulations for small $f$. For $\Delta \bar{\kappa}=$ const $<0$ (compare Fig. 5), Eq. (17) implies $\Delta \kappa / \Delta \bar{\kappa} \sim-f^{3 / 2-\nu}$, which becomes very large for large functionality.

The blob model calculation also gives some insight into the physical origin of the independence of the saddle-splay modulus per arm on the star functionality. As discussed after Eq. (15), the chains have to stretch for a spherical deformation of the membrane. However, in the case of a saddle deformation, the area available for the chains at any distance $r$ from the anchoring point remains completely unchanged. Therefore, the sizes and numbers of blobs are not affected by a saddle deformation, only their arrangement in space changes, which leaves the free energy unaltered.

\section{DISCUSSION AND CONCLUSIONS}

The interpretation of our results for the effective curvature elasticity is straightforward for symmetric diblock copolymers-like those investigated in balanced ternary microemulsions [18-20]—and for amphiphilic star polymers with equal numbers of hydrophobic and hydrophilic arms (of equal length) anchored to a membrane. In this case, there are identical polymer chains attached to the same anchoring point on both sides of the membrane [18]. Therefore, symmetry implies that the spontaneous curvature vanishes, and

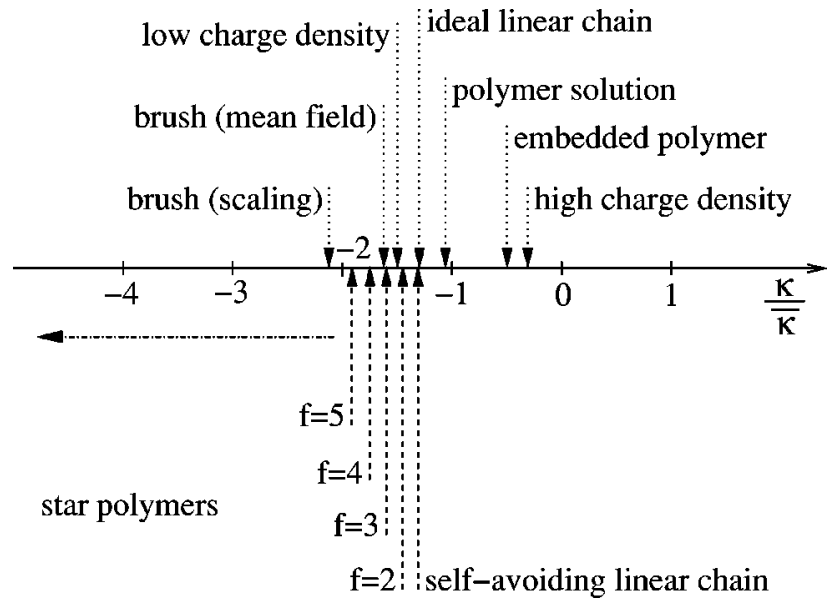

FIG. 6. Values of $\kappa / \bar{\kappa}$ for different systems $[2,3,5,11,12,14]$. Our results are indicated by dashed lines. Star polymers allow to access systematically a wide range of $\kappa / \bar{\kappa}$ ratios. As indicated by the arrow, in principle, the whole range of negative ratios is accessible.

our model to calculate the polymer effect on $\kappa$ and $\bar{\kappa}$ describes the system perfectly.

In the case of asymmetric block copolymers, or for endgrafted polymers-such as the PEO-lipids studied in bilayer vesicles [16] and lamellar phases [17] — the situation is more complicated. The pressure exerted on one side of the membrane by the polymer now induces a spontaneous curvature [4]. However, it also deforms the membrane locally into a conelike shape $[10,28,30-32]$. For star polymers, the conelike deformation increases with increasing functionality. The angle of the cone has been predicted from a blob model calculation to increase as $f^{3 / 2}$ [30].

In order to understand the generation of a spontaneous curvature for end-grafted chains, we have to distinguish two situations. The first is the case of a single linear or star polymer anchored to an infinite membrane, which becomes asymptotically flat far from the anchoring point, as studied in Refs. [10,28,30,31]. The membrane is found to be coneshaped in the area affected by the polymer, and takes a catenoid shape of zero mean curvature at larger distances. The integrated mean curvature in a perturbative calculation, in which the pressure distribution of the polymer anchored to a planar wall is used to determine the membrane shape, gives a value of $\sqrt{\pi / 6}\left(k_{B} T / 4 \kappa\right) R_{e}$ for ideal chains [31]. The second is a finite density of chains with grafting density $\sigma$. We can simply superimpose the shape deformations of the single chains and obtain a spontaneous curvature $\Delta c_{0}$ $=\sqrt{\pi / 6}\left(k_{B} T / 4 \kappa\right) \sigma R_{e}$ [31]. Remarkably, this is exactly the same result obtained by grafting ideal polymer chains onto a spherical surface, compare Eq. (3) and Table I. Thus, for ideal chains and sufficiently large bending rigidities - so that the pressure distribution is well approximated by the planar case-the local conelike deformation of the membrane shape does not affect the spontaneous curvature. This strongly supports the validity of our results also for asymmetric copolymers and for end-grafted linear and star polymers-possibly with small corrections.

It is important to note that the elastic moduli, which we 
calculate with our method, describe the effective curvature elasticity of the composite membranes on length scales, which are large compared to the size of an individual polymer mushroom.

The effect of star polymers on the membrane curvature elastic constants gives the unique possibility to vary the ratio $\kappa / \bar{\kappa}$ over a wide range, compare Fig. 6 . This provides the possibility to study the dependence of the behavior of mem- brane ensembles on the bending rigidity and saddle-splay modulus in more detail, and to control and tailor the properties of membrane systems.

\section{ACKNOWLEDGMENTS}

Stimulating discussions with E. Eisenriegler and D. Richter are gratefully acknowledged.
[1] W. Helfrich, Z. Naturforsch. C 28C, 693 (1973).

[2] K. Yaman, P. Pincus, F. Solis, and T.A. Witten, Macromolecules 30, 1173 (1997).

[3] S.T. Milner and T.A. Witten, J. Phys. (France) 49, 1951 (1988).

[4] R. Lipowsky, Europhys. Lett. 30, 197 (1995).

[5] C. Hiergeist and R. Lipowsky, J. Phys. II 6, 1465 (1996).

[6] C.M. Marques and J. Fournier, Europhys. Lett. 35, 361 (1996).

[7] S. Komura and S.A. Safran, Eur. Phys. J. E 5, 337 (2001).

[8] C. Hiergeist, V.A. Indrani, and R. Lipowsky, Europhys. Lett. 36, 491 (1996).

[9] Y.W. Kim and W. Sung, Phys. Rev. E 63, 041910 (2001).

[10] M. Breidenich, R.R. Netz, and R. Lipowsky, Eur. Phys. J. E 5, 403 (2001).

[11] E. Eisenriegler, A. Hanke, and S. Dietrich, Phys. Rev. E 54, 1134 (1996).

[12] A. Hanke, E. Eisenriegler, and S. Dietrich, Phys. Rev. E 59, 6853 (1999).

[13] H.N.W. Lekkerkerker, Physica A 159, 319 (1989).

[14] H.N.W. Lekkerkerker, Physica A 167, 384 (1990).

[15] R. Joannic, L. Auvray, and D.D. Lasic, Phys. Rev. Lett. 78, 3402 (1997).

[16] E. Evans and W. Rawicz, Phys. Rev. Lett. 79, 2379 (1997).

[17] H.E. Warriner et al., Science 271, 969 (1996).

[18] H. Endo et al., Phys. Rev. Lett. 85, 102 (2000).

[19] B. Jakobs et al., Langmuir 15, 6707 (1999).

[20] H. Endo et al., J. Chem. Phys. 115, 580 (2001).

[21] Y. Yang et al., Phys. Rev. Lett. 80, 2729 (1998).
[22] F. Castro-Roman, G. Porte, and C. Ligoure, Phys. Rev. Lett. 82, 109 (1999).

[23] N. Madras and A.D. Sokal, J. Stat. Phys. 50, 109 (1988).

[24] We have simulated polymers with 20 bonds for membranes curving both towards and away from the polymer, which provides partition functions for curvatures of both signs. We evaluate the membrane parameters for positive (negative) curvatures of (a) an ideal linear chain to be $a_{s p}=-0.1653$ $(-0.1654), \quad a_{\kappa}=0.1710(0.1714), \quad \bar{a}_{\kappa}=-0.1246(-0.1225)$, (b) a self-avoiding linear chain to be $a_{s p}=0.1574(0.1591)$, $a_{\kappa}=-0.1114(-0.1141), \quad \bar{a}_{\kappa}=-0.1533(-0.1537), \quad$ (c) a 4-armed star polymer to be $a_{s p}=0.9122(0.8918), a_{\kappa}=$ $-0.5189(-0.5549), \bar{a}_{\kappa}=-0.8624(-0.8624)$.

[25] B. Dünweg, D. Reith, M. Steinhauser, and K. Kremer, J. Chem. Phys. 117, 914 (2002).

[26] B. Li, N. Madras, and A.D. Sokal, J. Stat. Phys. 80, 661 (1995).

[27] M. Müller and G. Gompper, Phys. Rev. E 66, 041805 (2002).

[28] T. Bickel, C. Marques, and C. Jeppesen, Phys. Rev. E 62, 1124 (2000).

[29] M. Daoud and J.P. Cotton, J. Phys. (France) 43, 531 (1982).

[30] T. Bickel, C. Jeppesen, and C. Marques, Eur. Phys. J. E 4, 33 (2001).

[31] M. Breidenich, R.R. Netz, and R. Lipowsky, Europhys. Lett. 49, 431 (2000)

[32] A.R. Evans, M.S. Turner, and P. Sens, Phys. Rev. E 67, 041907 (2003). 